\title{
Emergence of diverse life cycles and life histories at the origin of multicellularity
}

\author{
Staps, Merlijn ; van Gestel, Jordi ; Tarnita, Corina E
}

\begin{abstract}
The evolution of multicellularity has given rise to a remarkable diversity of multicellular life cycles and life histories. Whereas some multicellular organisms are long-lived, grow through cell division, and repeatedly release single-celled propagules (for example, animals), others are short-lived, form by aggregation, and propagate only once, by generating large numbers of solitary cells (for example, cellular slime moulds). There are no systematic studies that explore how diverse multicellular life cycles can come about. Here, we focus on the origin of multicellularity and develop a mechanistic model to examine the primitive life cycles that emerge from a unicellular ancestor when an ancestral gene is co-opted for cell adhesion. Diverse life cycles readily emerge, depending on ecological conditions, group-forming mechanism, and ancestral constraints. Among these life cycles, we recapitulate both extremes of longlived groups that propagate continuously and short-lived groups that propagate only once, with the latter type of life cycle being particularly favoured when groups can form by aggregation. Our results show how diverse life cycles and life histories can easily emerge at the origin of multicellularity, shaped by ancestral constraints and ecological conditions. Beyond multicellularity, this finding has similar implications for other major transitions, such as the evolution of sociality.
\end{abstract}

DOI: https://doi.org/10.1038/s41559-019-0940-0

Posted at the Zurich Open Repository and Archive, University of Zurich ZORA URL: https://doi.org/10.5167/uzh-182977

Journal Article

Originally published at:

Staps, Merlijn; van Gestel, Jordi; Tarnita, Corina E (2019). Emergence of diverse life cycles and life histories at the origin of multicellularity. Nature Ecology and Evolution, 3(8):1197-1205.

DOI: https://doi.org/10.1038/s41559-019-0940-0 


\title{
Emergence of diverse life cycles and life histories at the origin of multicellularity
}

\author{
Merlijn Staps' ${ }^{1}$ Jordi van Gestel $\odot^{2,3,4,5 \star}$ and Corina E. Tarnita ${ }^{1 \star}{ }^{1 \star}$
}

\begin{abstract}
The evolution of multicellularity has given rise to a remarkable diversity of multicellular life cycles and life histories. Whereas some multicellular organisms are long-lived, grow through cell division, and repeatedly release single-celled propagules (for example, animals), others are short-lived, form by aggregation, and propagate only once, by generating large numbers of solitary cells (for example, cellular slime moulds). There are no systematic studies that explore how diverse multicellular life cycles can come about. Here, we focus on the origin of multicellularity and develop a mechanistic model to examine the primitive life cycles that emerge from a unicellular ancestor when an ancestral gene is co-opted for cell adhesion. Diverse life cycles readily emerge, depending on ecological conditions, group-forming mechanism, and ancestral constraints. Among these life cycles, we recapitulate both extremes of long-lived groups that propagate continuously and short-lived groups that propagate only once, with the latter type of life cycle being particularly favoured when groups can form by aggregation. Our results show how diverse life cycles and life histories can easily emerge at the origin of multicellularity, shaped by ancestral constraints and ecological conditions. Beyond multicellularity, this finding has similar implications for other major transitions, such as the evolution of sociality.
\end{abstract}

\section{T} he evolutionary history of life on Earth features multiple major transitions during which biological entities assembled into higher-order structures ${ }^{1-5}$. A key example of such a transition is the evolution of multicellular life from unicellular ancestors $^{6-9}$. Multicellularity has evolved repeatedly and is widespread across the tree of life, ranging from the canonical examples of multicellular organisms (animals, plants, fungi) to filamentous bacteria or aggregating social amoebae. Independent evolutionary origins of multicellularity have given rise to a wide variety of multicellular life cycles $^{9-18}$ that differ in their mode of group formation (how groups are built from single cells), the mode of group propagation or reproduction (how groups give rise to new groups), and in the resulting life history traits (for example, group size, longevity, or timing of group reproduction). For example, group formation can occur via cells staying together after division (ST, or clonal development), cells coming together (CT, or aggregation) or via a mix of CT and $\mathrm{ST}^{6,9,19,20}$, whereas group reproduction modes can range from binary fission (groups splitting into two) to the release of single-celled propagules or even the complete dissolution of groups into single cells $^{21-23}$. How diverse multicellular life cycles and life histories can come about remains an open question.

Our understanding of the life cycles that arise at the very origin of multicellularity has been greatly illuminated by recent empirical advances ${ }^{24-28,29}$. Experimental evolution studies have demonstrated that multicellular life cycles can readily emerge given the right preadaptations and ecological conditions $s^{30-34}$. Comparative genomics studies have shown that transitions to multicellularity are facilitated by the reorganization of molecular pathways that are already present in the unicellular ancestor, thus revealing the important role of the unicellular ancestor in shaping primitive multicellular life ${ }^{35-38}$. Despite these empirical developments, the emergence of multicellular life cycles from unicellular ancestors has received surprisingly limited theoretical attention. Instead, theoretical studies tend to take the emergence of a multicellular life cycle for granted (but see refs. ${ }^{39-41}$ ) and examine how different life cycles may be favoured under different ecological conditions ${ }^{19,21,42,43,44}$ or how group properties (such as group integrity or the division of labour between group members) may evolve in the context of a given life cycle ${ }^{45-50}$. There have been no theoretical studies that, starting from the unicellular ancestor, have systematically explored the multicellular life cycles and life histories that can originate.

Here, we approach this question by developing a mechanistic model for the evolution of multicellularity that explicitly accounts for the unicellular ancestor and its ecology (see Methods for full details and Table 1 for parameters). In this model, group formation results from cell stickiness, which we assume to arise as a promiscuous function of an ancestral gene. Evolution acts on the regulation of this gene, leading to the emergence of multicellular life cycles. Because the life cycle is an emergent feature of the model, this bottom-up approach allows us to explore, without a priori expectations, what life cycles may arise starting from a unicellular ancestor and how those life cycles are shaped by ecological conditions and ancestral constraints.

Specifically, we consider a finite population of haploid, asexually reproducing cells that undergo density-dependent population growth and experience two sources of mortality-one intrinsic and one due to predation. Since many unicellular organisms face fluctuating environmental conditions, such as the feast and famine cycles in soil-dwelling amoebae or the diurnal cycle in photosynthetic algae, we assume that our cells live in a fluctuating environment. For simplicity, we consider only two alternating environments of equal length. In each environment, selection favours (via a reproductive benefit) the expression of a different gene-gene $\mathrm{A}$ in environment 1 and gene B in environment 2. To allow selection to act on gene 
a

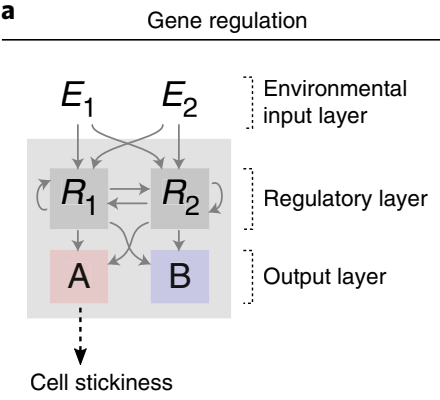

b

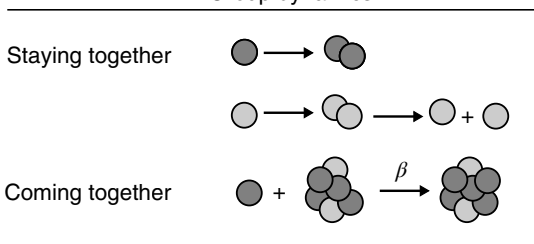

Cell release
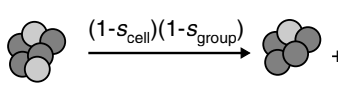

Sticky cell (A on)

Non-sticky cell (A off)
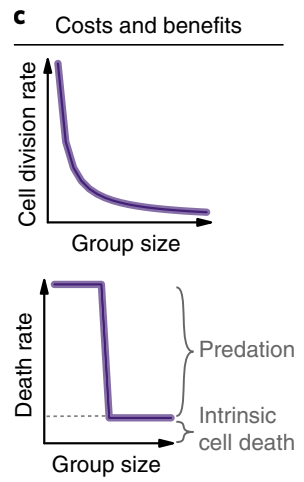

Fig. 1 | Overview of the model. a, Each cell is equipped with a gene regulatory network that senses the environment. $A, B$, $R_{1}, R_{2}$ denote genes; $E_{1}$ and $E_{2}$ denote environmental factors. Environment 1 is specified by $E_{1}=1, E_{2}=0$; environment 2 is specified by $E_{1}=0$, $E_{2}=1$. The optimal expression pattern in environment 1 is to express only gene $A$; the optimal expression pattern in environment 2 is to express only gene $B$. Deviations from these optimal expression patterns lead to a 70\% (if one gene has the incorrect expression status) or $90 \%$ (if both genes have the incorrect expression status) decrease in reproduction. Cell stickiness arises as a promiscuous function of gene A. b, Mechanisms of group formation and disintegration (see Methods for details and Table 1 for parameters). The probability to dissociate $\left(1-s_{\text {cell }}\right)\left(1-s_{\text {group }}\right)$ depends on cell stickiness $s_{\text {cell }}$ and group stickiness $s_{\text {group }}$ which is simply the mean stickiness of all group members. c, Costs and benefits of group formation. Cells in sufficiently large groups (size at least $k$ ) have a survival advantage because they incur no mortality due to predation.

regulation, each cell is equipped with a Boolean gene regulatory network $^{51-54}$ (Fig. 1a). Cells can sense their environment and, via gene regulation, determine the expression of genes $\mathrm{A}$ and $\mathrm{B}$. The gene regulatory network is subject to evolution: when a cell divides, the real-valued weights of the regulatory connections can change through mutation. Cell division, cell death, and mutations in the gene regulatory network all occur stochastically at each time step.

In each simulation, we first let the unicellular ancestor evolve in its fluctuating environment, starting from an empty regulatory network (that is, all network parameters are set to 0 ). A gene regulatory network arises that restricts expression of gene A to environment 1 and expression of gene B to environment 2 (Supplementary Fig. 1). Eventually, most cells in the population will be equipped with this phenotypic switch, although, because mutations continue to occur, other expression patterns will be present as well.

After a fixed number of time steps, chosen to be large enough for the phenotypic switch to have evolved in all simulations, we allow cells to form multicellular groups. Specifically, we assume that, due to ecological or physiological changes, the expression of gene A promiscuously gives rise to a rudimentary form of cell adhesion, in addition to its ancestral function ${ }^{55-57}$. This assumption is motivated by the fact that many independent transitions to multicellularity feature the co-option of ancestral genes for multicellular functions ${ }^{35-38,58-60}$. The ancestral gene A could, for instance, encode a cell surface protein that allows cells to interact with their external environment by binding to extracellular entities ${ }^{61}$; this surface protein could then 'accidentally' start causing cells to stick to each other as well. Since stickiness arises as a promiscuous trait of an ancestral protein, we do not expect that becoming sticky is directly costly to the cell (although there will be indirect costs arising from group formation, as detailed below). We first consider the case where all cells expressing gene A have the same constant level of stickiness $s$ between 0 and 1; subsequently, we also investigate how the ability to evolve the level of stickiness impacts our results. Cells that do not express gene A are not sticky.

At first, we assume that groups can only form when dividing cells fail to separate after division (ST) (Fig. 1b); subsequently, we also explore aggregation (CT) as an additional group formation mechanism. Once formed, groups can also disintegrate. For simplicity, we assume that group disintegration occurs as a result of single cells detaching one by one, and not via fragmentation into larger subgroups. The probability of detachment depends on the stickiness levels of both the cell and the group, with sticky cells less likely to detach and stickier groups less likely to lose cells (Fig. 1b).

Ecologically, there are both costs and benefits to group formation (Fig. 1c). We assume that groups that have grown sufficiently large ( $\geq k$ cells) have a survival advantage, for example because they are able to escape predation by a phagotrophic predator ${ }^{34,62}$. Thus, groups that are below size $k$ are subject to the same predation mortality as single cells, while groups of at least size $k$ incur no mortality due to predation. All cells are subject to intrinsic cell death, whether they are in a group or not. At the same time, we assume that group formation comes at a reproductive cost to constituent cells, to account for the fact that increased local cell density may lead to limited access to nutrients and accumulation of waste products ${ }^{63}$. These costs are implemented by dividing each cell's probability to reproduce by the size of the group it belongs to. The purpose of these large costs is to consider a 'worst-case' scenario for the evolution of multicellularity. Because the costs of group formation increase with group size, groups cannot grow indefinitely. The maximum size that groups can reach is determined by cell stickiness, with stickier cells being able to build larger groups (Supplementary Analysis 1 and Supplementary Fig. 2).

We use our model to simulate the origin of multicellularity and investigate the properties of the emergent multicellular life cycle that derives from the temporal expression pattern of gene A (which determines when cells are sticky and can engage in group formation). Two selective forces act simultaneously on the regulation of gene A: selection on the ancestral function favours cells that restrict expression of gene A to environment 1 , whereas selection on the promiscuous stickiness function may-due to the costs and benefits associated to multicellularity-favour cells with alternative expression patterns. We use the term strategy to refer to the temporal expression pattern of gene A (Supplementary Methods); for example, the ancestral strategy (henceforth strategy I) is the one that expresses gene A only in environment 1 .

Diverse life cycles emerge during the evolution of multicellularity. We study different ecological scenarios for the evolution of multicellularity, by varying cell stickiness $s$ (which determines how large groups can grow; Supplementary Fig. 2) and the minimum group size $k$ required to avoid predation (which determines how large groups need to be for multicellularity to be beneficial). We ran simulations for a range of parameter combinations and for each 


\begin{tabular}{|c|c|c|}
\hline Parameter & Interpretation & Value \\
\hline k & $\begin{array}{l}\text { Minimum group size required to avoid predation } \\
\text { (varied across simulations) }\end{array}$ & \\
\hline s & Cell stickiness (varied across simulations) & \\
\hline$\beta$ & Rate of coming together (only when CT is included) & 0.005 \\
\hline N & $\begin{array}{l}\text { Carrying capacity (maximum number of cells in } \\
\text { the population) }\end{array}$ & 500 \\
\hline$T$ & $\begin{array}{l}\text { Duration of an environmental period (in time } \\
\text { steps); twice the number of time steps spent in } \\
\text { either environment }\end{array}$ & 600 \\
\hline$T_{1}$ & $\begin{array}{l}\text { Duration of ancestral evolution, before } \\
\text { multicellularity is possible (in time steps) }\end{array}$ & $10^{5}$ \\
\hline$b_{\max }$ & $\begin{array}{l}\text { Division rate of a cell with the correct expression } \\
\text { pattern for its current environment }\end{array}$ & 0.1 \\
\hline$b_{\text {int }}$ & $\begin{array}{l}\text { Division rate of a cell if the expression of one of } \\
\text { the two genes deviates from the optimal } \\
\text { expression pattern }\end{array}$ & 0.03 \\
\hline$b_{\min }$ & $\begin{array}{l}\text { Division rate of a cell if the expression of both } \\
\text { genes deviates from the optimal expression pattern }\end{array}$ & 0.01 \\
\hline$d_{\text {cell }}$ & Intrinsic cell death rate & 0.001 \\
\hline$d_{\text {pred }}$ & Predation rate & 0.015 \\
\hline$\mu$ & Mutation rate & 0.01 \\
\hline$\sigma_{\mathrm{GRN}}$ & $\begin{array}{l}\text { Standard deviation of mutations in the gene } \\
\text { regulatory network }\end{array}$ & 0.3 \\
\hline$\sigma_{\text {sticky }}$ & $\begin{array}{l}\text { Standard deviation of mutations in cell stickiness } \\
\text { (only when stickiness is evolvable) }\end{array}$ & 0.15 \\
\hline$h_{x}$ & $\begin{array}{l}\text { Activation threshold of gene } X \text { in the gene } \\
\text { regulatory network (evolvable, within }[-1,1] \text { ) }\end{array}$ & \\
\hline$w_{X \rightarrow Y}$ & $\begin{array}{l}\text { Weight of a connection from node } X \text { to node } Y \text { in the } \\
\text { gene regulatory network (evolvable, within }[-1,1] \text { ) }\end{array}$ & \\
\hline
\end{tabular}

All rates are implemented in the model as probabilities per unit time step. For birth rates, density dependence and group size are taken into account: to obtain the effective birth rate, the birth rate presented in the table is multiplied by $\left(1-\frac{n}{N}\right) /$ where $n$ is the current population size and $j$ is the group size of the cell in question. Values are omitted for parameters that are varied across simulations $(k, s)$ and evolvable parameters of the gene regulatory network $\left(h_{x}, w_{x \rightarrow y}\right)$.

simulation we determined the most prevalent strategy at the end of the simulation (Fig. 2a). This strategy may either outcompete other strategies completely or be the most abundant one of two coexisting strategies (for example, Fig. 2b).

For large $k$, the expected benefits of multicellularity are low regardless of the value of $s$ (since, even for high values of $s$, it is unlikely that groups will reach size $k$ ). If $s$ is low, the costs of group formation are also low and cells retain the ancestral strategy (Fig. 2a,b): groups form in environment 1 but fall apart instantaneously upon entering environment 2, where cells lose their stickiness (Fig. $2 \mathrm{c}$, strategy I). At higher values of $s$, cells incur larger reproductive costs due to group formation, while still receiving little expected benefit in return. Consequently, selection acts against multicellularity and favours cells that avoid the costs of group formation by reducing the expression of gene A. Indeed, in this parameter regime, we observe the evolution of a second strategy, II, in which gene A is still only expressed in environment 1 , but in an oscillating fashion (Fig. 2a,b). This innovation counteracts group formation-because groups that have formed will fall apart as soon as cells lose their stickinesswhile, at the same time, mitigating the costs associated with failure to express gene $\mathrm{A}$ in environment 1 . The resulting life cycle is essentially unicellular (Fig. 2c).

For lower values of $k$, the selective landscape is different, because the survival advantage of multicellularity can come into play, provided that cells are sufficiently sticky for groups to consistently reach size $k$. Indeed, when $s$ is sufficiently large, a third strategy, III, emerges, in which cells express gene A constitutively (Fig. 2a,b). Strategy III gives rise to a life cycle with obligate group formation (Fig. 2c), in which cells are only solitary from the moment they accidentally detach from their group until their next cell division. Strategy III either replaces the ancestral strategy completely or coexists with it (Fig. 2b). Coexistence of strategies I and III is facilitated by the environmental fluctuations; altering the frequency of environmental fluctuations disrupts the coexistence (Supplementary Analysis 2 and Supplementary Figs. 3 and 4).

When $s$ is too high, cells rarely detach from their group, which affects group propagation and leads to unnecessarily large (and thereby reproductively costly) groups. As a consequence, when $s$ is increased at intermediate values of $k$-where strategies I and III coexist-strategy III loses ground to strategy I. When $s$ is increased at the smallest values of $k$, an unexpected strategy IV appears, which expresses gene $\mathrm{A}$ in both environments except at the shift from environment 2 to environment 1 (Fig. 2a,b). The brief interruption of stickiness causes groups that have formed to fall apart into solitary cells, leading to a life cycle that features a fixed period of group growth, followed by a reproduction event in which all cells in the group are released as unicellular propagules (Fig. 2c). Thereby, strategy IV simultaneously solves both problems of unlikely propagation and unnecessarily large groups.

The emergence of four different strategies, I-IV, is robust to changes in the frequency of the environmental fluctuations, the costs and benefits associated to multicellularity, and the evolutionary history of the unicellular ancestor (Supplementary Analysis 3 and Supplementary Figs. 4 and 5), although the parameter regimes under which the different strategies emerge may be altered. For example, in a faster-changing environment, strategies I and IV, for which the environment dictates how much time groups have available to grow, emerge under a narrower range of conditions (Supplementary Fig. 4).

The evolution of novel life cycles depends on changes in the ancestral gene regulatory network. To assess these changes, we qualitatively analysed the networks that emerged in the simulations displayed in Fig. 2. In this analysis, we restrict attention to critical network connections and ignore redundant connections that can be removed without affecting network function (see Supplementary Analysis 4 for details). We find that the emergent networks encoding strategies I and III can be classified into eight qualitatively different network topologies, consisting of only two or three network connections (Fig. 3). For the more complex strategies II and IV, a wider range of network topologies is realized, as these strategies require more network connections-including connections within the regulatory layer (Fig. 3). Accordingly, when we run simulations in which the ancestral gene regulatory network is simplified by removing one of the regulatory nodes, evolution of strategies II and IV is affected, with strategy IV failing to evolve altogether (Supplementary Fig. 6a; adding an extra regulatory node, however, does not lead to the evolution of additional strategies; see Supplementary Fig. 6b). Thus, the evolution of novel life cycles is constrained by the complexity of the ancestral gene regulatory network.

Finally, we relaxed the assumption that cell stickiness is a rigid physiological constraint and investigated the emergence of multicellular life cycles when evolution acts not only on the regulation, but also on the level of cell stickiness. Cell stickiness is initialized at 0 and has to arise gradually via cumulative mutations (see Methods). Cells still need to express gene A to be sticky, but two cells that express gene A can now evolve different stickiness levels. In this evolvable-stickiness scenario, we recover the evolution of the multicellular strategies III (for $k=3$ ) and IV (for $k=2$ ) (Supplementary Fig. 7). For $k \geq 4$ stickiness stays close to 0 and cells retain the ancestral strategy I (see Supplementary Fig. 7 for $k=4$ ). 


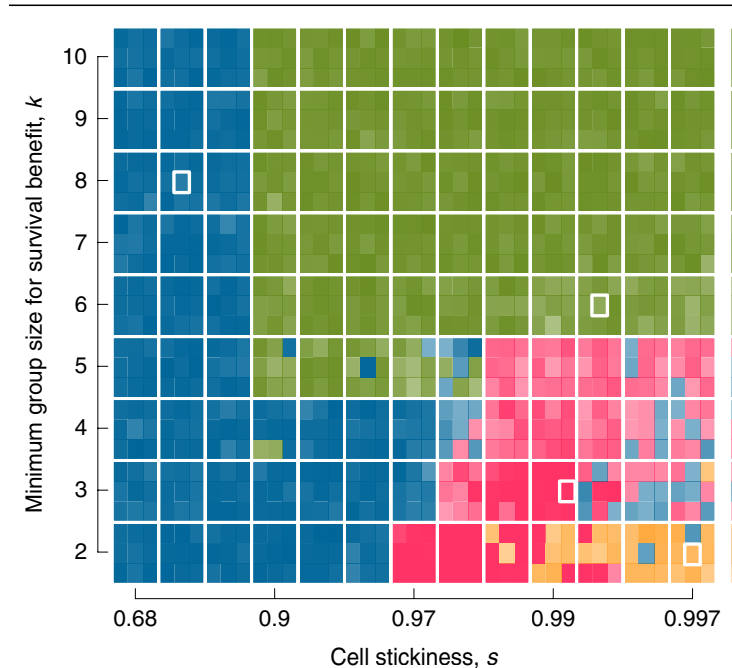

b

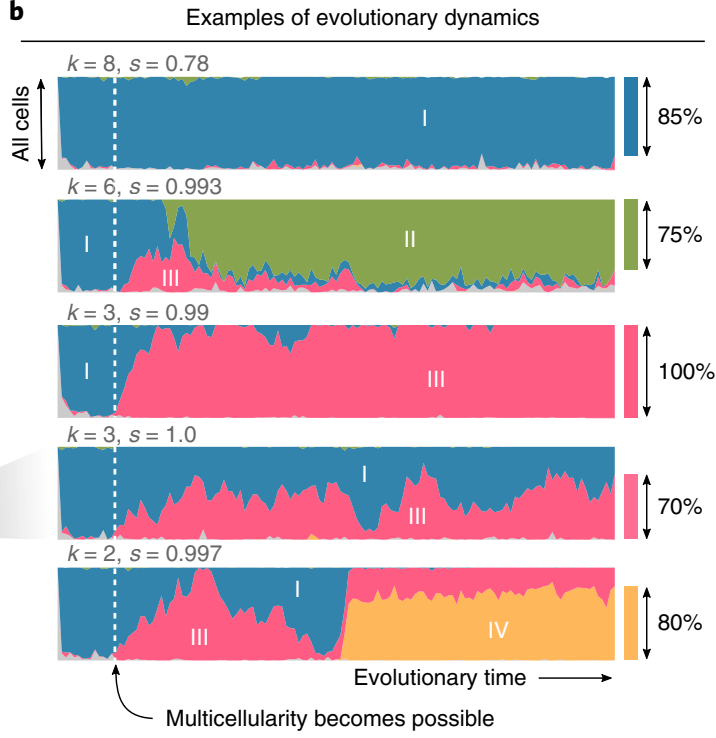

Multicellularity becomes possible

Emergent life cycles

I:

only in environment 1

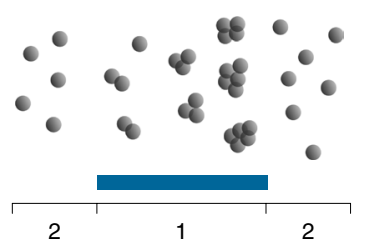

II

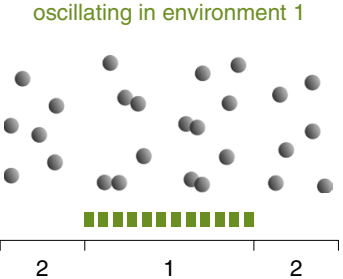

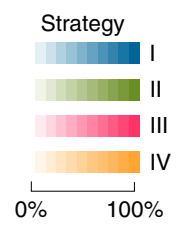

Fraction of cells

in final population

adopting strategy

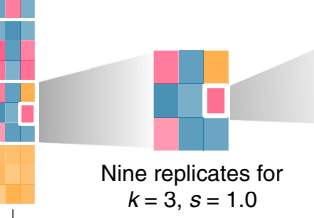

: always

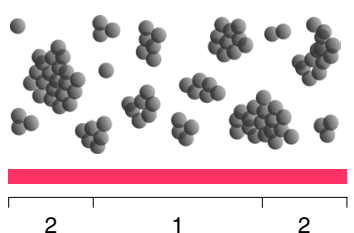

IV:
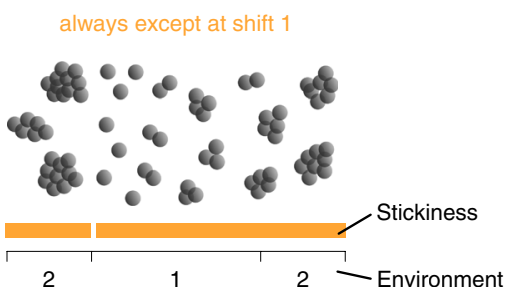

Fig. 2 | Emergence of diverse multicellular life cycles. a, Results of evolutionary simulations. Colour indicates the most prevalent strategy at the end of the simulation; colour intensity indicates what fraction of the final population adopts the most prevalent strategy (see $\mathbf{b}$ for examples). For each parameter combination (large rectangles), results are shown for nine replicate simulations (small sub-rectangles). Four strategies are distinguished on the basis of the expression of gene A: only in environment 1 (strategy I), only in environment 1 but in an oscillating fashion (strategy II; the period of the oscillations can be two, three or four time steps), always (strategy III), or always except at the shift from environment 2 to environment 1 (strategy IV). The stickiness range on the $x$-axis is chosen such that the probability $(1-s)^{2}$ for sticky cells to dissociate from a group ranges from $10^{-1}$ (for $s=0.68$ ) to $10^{-5}$ (for $s=0.997$ ) (all other parameters as in Table 1). When $s=1$, sticky cells never dissociate. $\mathbf{b}$, Evolutionary dynamics for five simulations with qualitatively different behaviours, corresponding to the sub-rectangles highlighted with a white contour in $\mathbf{a}$. Colours indicate strategies; strategies other than I-IV are depicted in grey. Each simulation is run for $10^{6}$ time steps (1,666 environmental periods), with multicellularity being possible from $t=T_{1}=10^{5}$ (see Supplementary Fig. 1 for unicellular evolutionary dynamics). Percentages and colours indicate the most prevalent strategy and its prevalence (see a). c, Different strategies lead to different emergent life cycles. Schematics give a qualitative description of the life cycle over one environmental period (from environment 2 to environment 1 and back); quantitative aspects of the life cycle (for example, attained group sizes) also depend on the values of $k$ and s.

Strategy II never evolves, because disadvantageous group formation can now be prevented by lowering stickiness rather than by downregulating the expression of gene A. Interestingly, the stickiness level that a cell evolves depends on its strategy (Supplementary Fig. 7). Cells that are continually sticky (strategy III) do not evolve maximum stickiness, ensuring that groups do not grow unnecessarily large and propagules still occasionally detach from their group. In contrast, evolution of strategy IV is coupled to the evolution of maximum stickiness. In this case, cells never detach accidentally, so group dissolution is the only way that groups can reproduce. Thus, the evolution of a specific multicellular life cycle is coupled to the evolution of a certain cell stickiness level.

Emergent life cycles vary in their life history traits. The nascent life cycle determines the life history traits of the emergent groups, including group size, group longevity, and the various aspects of group reproduction (efficiency, timing, and so on). To compare the life history traits of different emergent life cycles, we focused on an ecological regime in which two multicellular strategies (III and IV) have a selective advantage over the ancestral strategy. This allows us to compare the resulting life cycles under identical ecological conditions (Fig. $4 ; k=2$ and $s=0.99$ ).

We quantified group life history traits by tracking individual groups from the moment they were formed by division of a solitary cell until they completely disintegrated by a combination of cell death and cell detachment (there is no group death for $k=2$ ). Relative to strategy IV, strategy III leads to larger (Fig. 4a) and longer-lived (Fig. 4b) groups, more variable group lifespan (Fig. 4b), as well as more variable number of offspring per group (that is, the total number of unicellular propagules released over the group lifespan) (Fig. 4c). To explore the efficiency of group propagation, we tracked the fate of individual cells. Whereas in strategy III groups only $9 \%$ of cells are eventually released (with the other $91 \%$ staying in the group until they undergo intrinsic cell death), in strategy IV groups this proportion increases to 59\% (Fig. 4d), indicating that strategy IV groups are able to propagate more efficiently.

Thus, despite the fact that there is only a small difference in gene expression between strategies III and IV, they give rise to life cycles 

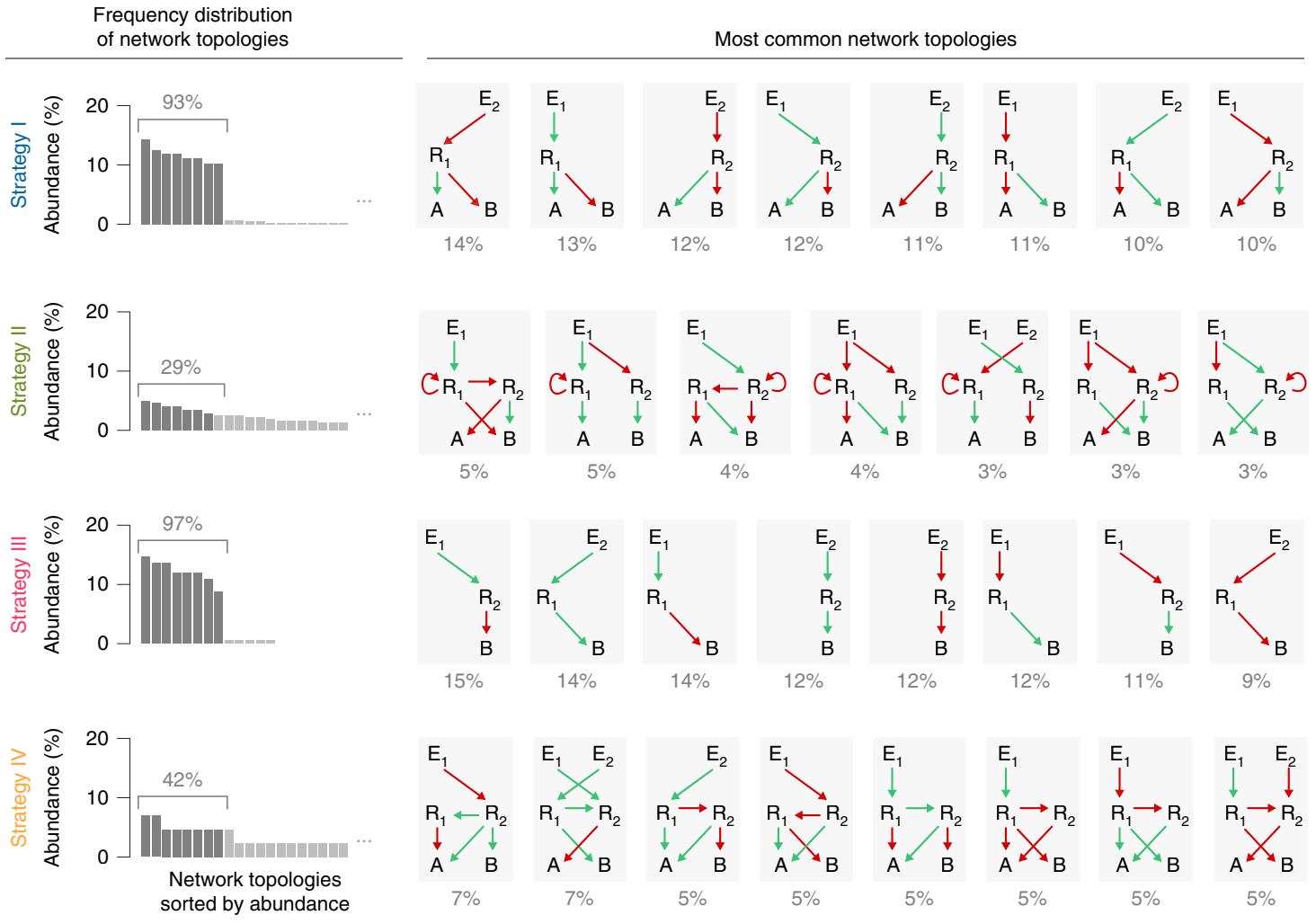

Fig. 3 | Topologies of evolved gene regulatory networks. Gene regulatory networks were abstracted to network topologies by only taking into account the connections critical for network function (see Supplementary Analysis 4). Each of strategies I-IV can be encoded by different network topologies. Bar charts show the frequency distribution of different network topologies encoding each strategy, computed across all simulations displayed in Fig. 2a in which the strategy evolves. Percentages indicate the combined frequency of the eight most common network topologies. For each strategy, the most common network topologies (corresponding to darker bars) are displayed, in order, on the right. Percentages below network topologies indicate the fraction of simulations in which each topology arises. Stimulatory interactions (positive connection weights) are in green; inhibitory interactions (negative connection weights) are in red. Only networks for which the expression of gene B is restricted to environment 2 are included.
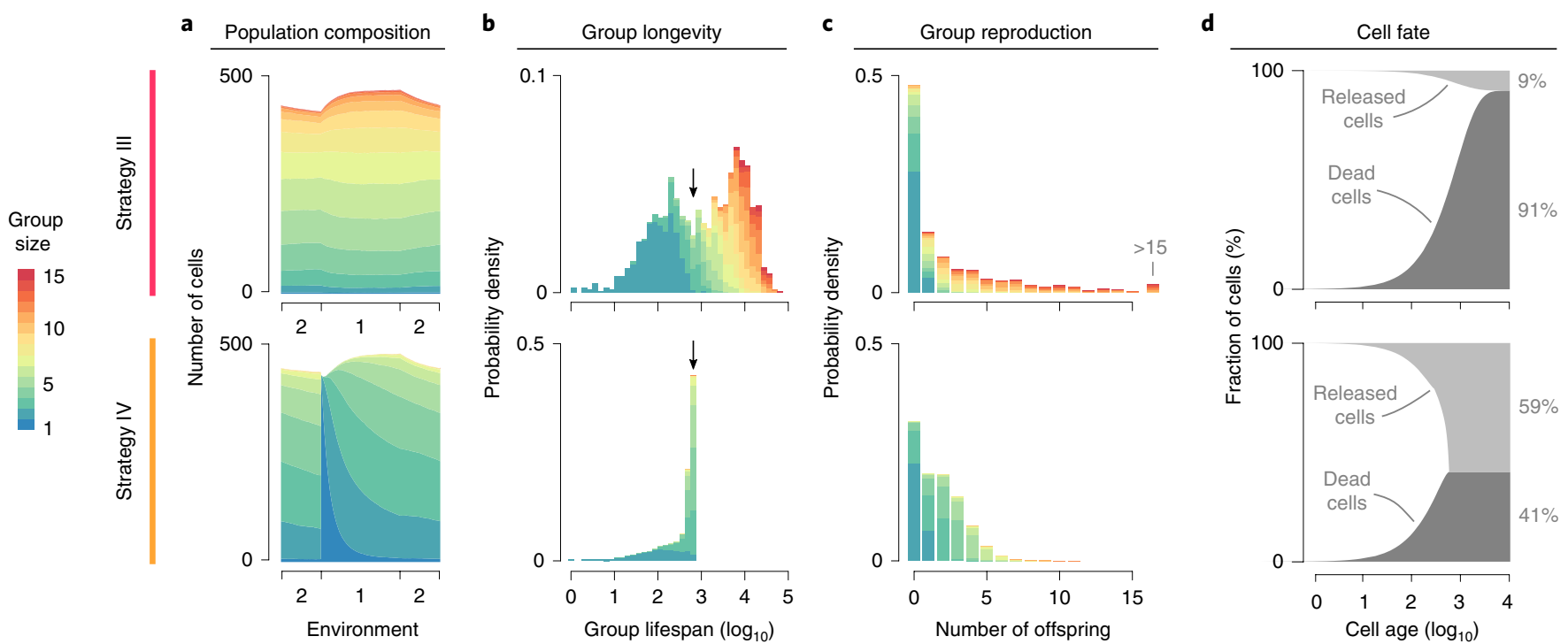

Fig. 4 | Emergent multicellular life cycles differ in their life history strategies. Life histories of strategy III (top row) and strategy IV (bottom row) for $k=2$ and $s=0.99$, grown in isolation and in the absence of mutation (remaining parameters as in Table 1). a, Population composition over one environmental period. Colour indicates group size. $\mathbf{b}$, Distribution of group lifespan. Arrows indicate the length of one environmental period. $\mathbf{c}$, Distribution of the number of offspring per group (that is, the number of cells released over the lifespan of the group). For $\mathbf{b}, \mathbf{c}$, groups are tracked from the moment they are born as a 2-cell until the moment they have disintegrated completely by a combination of cell death and cell release. Total number of groups is 1,488 for strategy III and 33,558 for strategy IV. Colour indicates the maximum size a group reaches over its lifespan (same colours as in a). d, Fate of individual cells. Cells are tracked until they are released from their group or have undergone intrinsic cell death. Total number of cells is 38,481 for strategy III and 73,782 for strategy IV. 
a

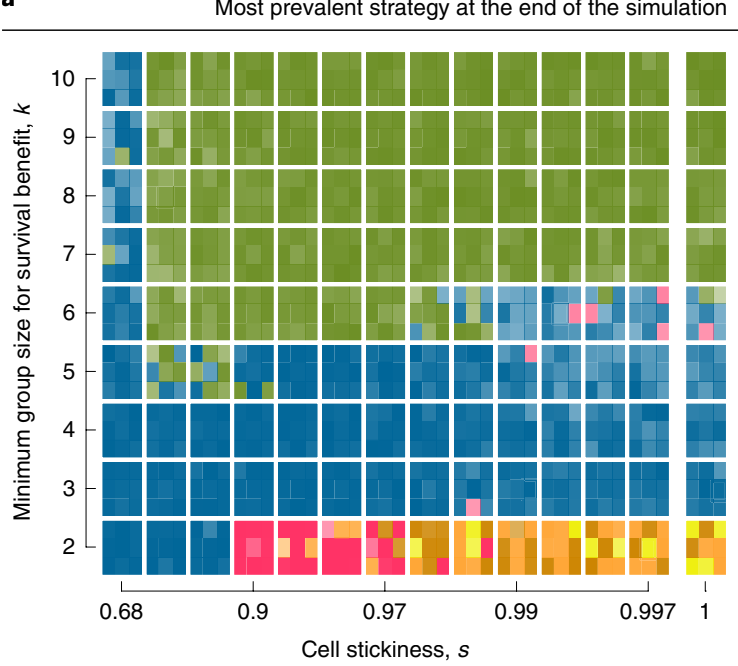

b

vis

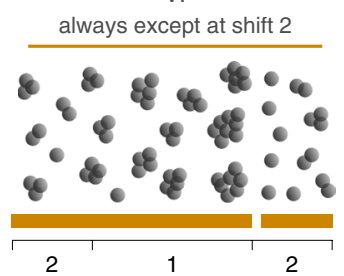

Emergent life cycles

$\mathrm{VI}:$

always except at both shifts

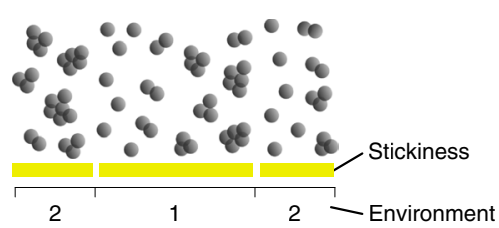

Long-term evolutionary dynamics $(k=2, s=0.99)$

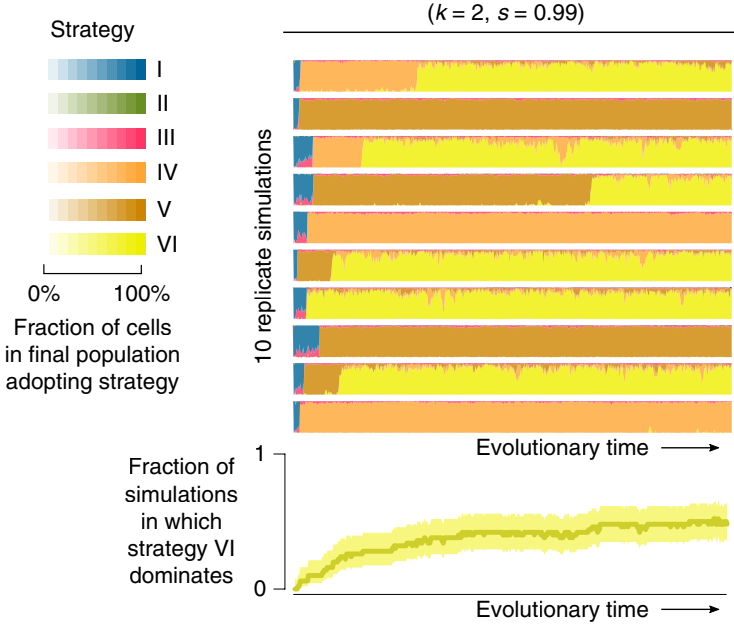

d

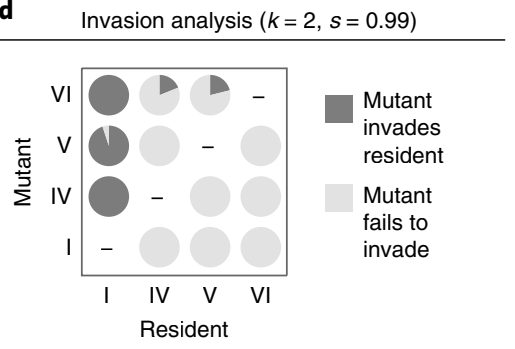

Fig. $\mathbf{5}$ | Coming together facilitates the evolution of novel life cycles. a, Results of evolutionary simulations when CT is possible as an additional group formation mechanism (see Table 1 for parameter values). Colour indicates the most prevalent strategy at the end of the simulation; colour intensity indicates what fraction of the final population adopts the most prevalent strategy. For each parameter combination (large rectangles), results are shown for nine replicate simulations (small sub-rectangles). With strategy $V$, cells are always sticky except at the shift from environment 1 to environment 2; with strategy $\mathrm{VI}$, cells lose their stickiness at both environment shifts (strategies I-IV are as in Fig. 2). Each simulation is run for $10^{6}$ time steps (1,666 environmental periods). b, Life cycle schematics for strategies $V$ and $V I$. c, Long-term evolutionary dynamics for $k=2$, $s=0.99$. Population composition over time shown for ten representative simulations (colour indicates strategy; see $\mathbf{a}$ ). Simulations are run 16 times longer than in $\mathbf{a}$; the $x$-axis spans $16 \times 10^{6}$ time steps. The fraction of simulations in which strategy VI dominates is computed over 50 replicate simulations. The depicted $95 \%$ confidence interval was computed using Wilson's method ${ }^{90}$. d, Invasion analysis for $k=2, s=0.99$. Mutants are initiated at $10 \%$ abundance and are said to invade if their abundance has increased after $2.4 \times 10^{5}$ time steps (when in many cases only one strategy remains). Each pie chart summarizes results for 100 replicate invasion experiments.

with drastically different life history traits. Whereas strategy IV leads to small, short-lived groups that reproduce quickly in essentially a single reproductive event (akin to r-selected species ${ }^{64,65}$ ), strategy III yields larger, longer-lived groups that reproduce repeatedly (akin to K-selected species ${ }^{64,65}$ ).

Group formation via CT favours short life cycles. Thus far, we studied a scenario in which groups can only form via ST. Depending on ecological conditions, CT can also occur, as cells 'bump into' groups and become attached. We implemented CT in addition to ST, assuming a well-mixed environment where solitary cells interact randomly with each other and with groups (Fig. 1b). CT allows for the formation of heterogeneous groups; however, we do not expect within-group conflicts to play a big role in our model, because group members that try to free-ride by losing their stickiness (to avoid the cost of expressing gene $\mathrm{A}$ in the wrong environment) are quickly released from their group. Nevertheless, CT has a potential to affect the evolutionary dynamics, because it allows for fast group formation ${ }^{66,67}$.

Indeed, upon introducing CT, two new strategies, V and VI, emerge, in addition to the four found previously (Fig. 5a). Strategy $\mathrm{V}$ is the 'mirror image' of strategy IV: cells lose their stickiness upon entering environment 2 instead of environment 1 (Fig. 5b). With strategy VI, cells lose their stickiness at both environment shifts
(Fig. 5b). Strategies V and VI are unique among strategies I-VI in that they depend on the formation of groups from single cells in environment 2. Group formation via ST is slower in environment 2 than in environment 1 , due to the reproductive costs associated with expressing gene A outside its ancestral range. When CT is possible, however, group formation can occur rapidly in either environment, which explains why strategies V and VI only evolve in this case.

The two new strategies, V and VI, arise for low $k$ and sufficiently high $s$. Dynamically, we observe a succession of strategies in this range: initially one of the strategies IV and V emerges, to be eventually replaced by strategy VI (Fig. 5c). It is a matter of chance which one of strategies IV and V initially emerges in a given simulation; however, once one of them has emerged, it excludes the other (Fig. 5d). Strategy VI is able to invade both strategies IV and V (Fig. 5d), but it can take a long time to emerge (Fig. $5 \mathrm{c}$ ) because networks encoding strategy VI are rare (Supplementary Fig. 8). Strategy VI is evolutionarily stable (Fig. 5d), resisting invasion by other strategies. Because strategy VI leads to a twofold shorter life cycle than strategies IV and $\mathrm{V}$-the maximum group lifespan is halved and reproduction via complete dissolution occurs twice as frequently (Fig. 5b) - these results suggest that CT favours shorter life cycles.

Indeed, comparing Fig. 5a to Fig. 2a shows that when CT is possible, shorter life cycles (I, IV, V, VI compared with III) evolve under a 
wider range of conditions. Frequently reverting to the solitary stage allows cells to avoid the reproductive costs associated with forming unnecessarily large groups; when CT is possible, solitary cells can then reassemble into groups of beneficial sizes faster than with ST alone. In the resulting life cycles, $\mathrm{CT}$ is responsible for the quick assembly of small groups from single cells, whereas ST facilitates continued group growth when small groups have already formed and few solitary cells remain (Supplementary Fig. 9).

\section{Discussion}

Taken together, our results demonstrate how diverse life cycles and life histories could emerge at the origin of multicellularity through the co-option of ancestral regulatory mechanisms. Our theoretical approach, which explicitly accounts for the internal organization of the unicellular ancestor and its ecology, allows us to untangle some of the factors shaping nascent life cycles, including the physiological constraints on group formation, the available environmental information that can be used to scaffold the life cycle, and the regulatory complexity of the ancestor. However, even though we include these various layers of complexity, we have kept our model purposefully simple, reducing the input that cells receive to global, periodic environmental information, limiting the group formation mechanisms to either only ST or a combination of CT (only via solitary cells) and ST, and disregarding spatial structure and group geometry. Including additional biological features would further add to our understanding of the life cycles that can emerge during a transition to multicellularity. For example, if groups have an explicit geometry ${ }^{68}$, the likelihood of within-group conflicts could increase as free-riding, non-sticky cells might get trapped inside the group (rather than be very quickly expunged) and grow at a much faster rate than the rest ${ }^{69}$. Similarly, an interesting possibility arises if, in addition to the global environment, cells also have the ability to sense local cues, such as the state of their current group (for example, size, age, composition), and adapt their behaviour accordingly. Such innovations could lead to the evolution of developmental programs that establish a life cycle without relying on environmental triggers ${ }^{25,26,70-74}$.

There are clear parallels between the primitive life cycles in our model and the life cycles of extant multicellular organisms, although we stress that these comparisons should be made cautiously since our model focuses on life cycles that arise at the very origin of multicellularity. Our life cycles with obligate group formation, in which groups are long-lived and reproduce through the repeated production of single-celled propagules, resemble the life cycles of both the paradigmatic examples of multicellularity (animals, plants, fungi), and of some more primitive multicellular entities, for example, bacterial biofilms ${ }^{75,76}$. The life cycles with short-lived groups that propagate only once, by simultaneously releasing all cells that make up the group, are reminiscent of fruiting body formation in cellular slime moulds and myxobacteria. Interestingly, our model predicts that this type of life history is particularly favoured when $\mathrm{CT}$ is available as a group formation mechanism, which is consistent with the fact that most examples of aggregative multicellularity are indeed characterized by a short-lived multicellular life stage with a single propagation event ${ }^{77-79}$. Notably, this outcome results from our model even in the absence of within-group conflicts, which are typically considered to be responsible for the transience of CT life cycles ${ }^{25,26}$.

Although we focused on multicellularity, our approach is sufficiently general to have implications for other major hierarchical transitions as well, such as the evolution of sociality ${ }^{3,4,80-82}$. Our work suggests that, analogous to multicellular life cycles, diverse grouping behaviours in social animals ${ }^{83-89}$ could have evolved early during evolutionary transitions to sociality, drawing on phenotypic properties and decision-making strategies that were already present in the solitary ancestor.

\section{Methods}

For the evolutionary simulations, a computational model was implemented in Python (see Supplementary Methods for pseudocode). All relevant parameters are in Table 1 .

Gene regulation. Each cell is equipped with a gene regulatory network (GRN) that is subject to evolution (Fig. 1a). The GRN consists of three layers: an input layer, a regulatory layer and an output layer. The input layer consists of two external factors $E_{1}$ and $E_{2}$ that represent environmental input. Environment 1 is specified by $\mathrm{E}_{1}=1$ and $\mathrm{E}_{2}=0$; environment 2 is specified by $\mathrm{E}_{1}=0$ and $\mathrm{E}_{2}=1$. The environment switches back and forth between environment 1 and environment 2 every $T / 2$ steps. Thus, an environmental period consists of $T$ time steps during which the population experiences each environment for $T / 2$ time steps. The regulatory layer consists of two genes $R_{1}, R_{2}$ that can respond to environmental input and control the expression of downstream genes $\mathrm{A}$ and $\mathrm{B}$ in the output layer. Expression values are Boolean, that is, at each time step each gene is either 'off' (0) or 'on' (1). We use a synchronic updating rule in which a gene $Y$ is switched on if the input it receives exceeds its activation threshold $h_{Y}$. Formally, gene $Y$ is expressed at time $t$ if $\sum_{Z} w_{Z \rightarrow Y} Z(t-1)>h_{Y}$, where the sum runs over all inputs $Z$ of $Y, w_{Z \rightarrow Y} \in[-1,1]$ is the weight of the connection between $Z$ and $Y$, and $Z(t-1)$ is the expression of $Z$ at time $t-1$. If $w_{Z \rightarrow Y}$ is positive, $Z$ is said to stimulate the expression of $Y$; if negative, $Z$ is said to inhibit the expression of $Y$. There are 12 possible network connections: four connections from the input layer to the regulatory layer, four connections within the regulatory layer, and four connections from the regulatory layer to the output layer. In total, the model contains 16 evolvable parameters (12 interaction weights and four activation thresholds), which are all initialized at 0 and are allowed to evolve independently. Mutations in the gene regulatory network occur when cells divide. During division, each connection weight and threshold value is mutated with probability $\mu$. Mutations are implemented by adding to the value of the mutated element a random value drawn from the normal distribution with mean 0 and standard deviation $\sigma_{\mathrm{GRN}}$. If the resulting value lies outside $[-1,1]$, the extreme value that is exceeded is taken as the new value.

Reproduction and death. At each time step, each cell has a certain probability to divide and a certain probability to die. The division probability of a cell depends on its phenotype (that is, whether it expresses genes $\mathrm{A}$ and $\mathrm{B}$ ) and the environment. In environment 1 , cells with the optimal phenotype (A on, $B$ off) divide with probability $b=b_{\max }$. Cells with the opposite phenotype (A off, B on) divide with a much lower probability $b=b_{\min }$. Cells with other phenotypes (A and B both on, or $\mathrm{A}$ and $\mathrm{B}$ both off) divide with an intermediate probability $b=b_{\text {int }}$. In environment 2 the situation is exactly the opposite, and the maximum division probability is achieved by cells expressing gene B but not gene A. We implement density dependence by letting the population grow logistically with carrying capacity $N$. This means that, at any given time, each cell's division probability $b$ is multiplied by $1-n / N$, where $n$ is the number of cells at that time. There are two sources of mortality. The main contributor to mortality is predation, which occurs with probability $d_{\text {pred. }}$. There is also intrinsic cell death, occurring with a much smaller probability $d_{\text {cell }}$. In contrast to cell division, death occurs independently of a cell's phenotype.

Stickiness. We first let the unicellular ancestor evolve for $T_{1}$ time steps, without multicellularity being possible. After these $T_{1}$ time steps, cells expressing gene A become sticky; all such cells have the same stickiness $0 \leq s \leq 1$. Cells that do not express gene A are not sticky. We also consider a variant of the model in which stickiness itself is evolvable (while still being linked to expression of gene A). In this case, initially all cells have stickiness 0 . After the first $T_{1}$ time steps, mutations in stickiness occur with probability $\mu$ when cells divide. For simplicity, we use the same mutation probability as for the GRN. Similar to mutations in the GRN, mutations in stickiness are implemented by adding to the current stickiness a random value drawn from the normal distribution with mean 0 and standard deviation $\sigma_{\text {sticky }}$. If the new stickiness lies outside $[0,1]$, we take the extreme value that was exceeded as the new value.

Group dynamics. Group formation via ST occurs whenever a cell divides (Fig. 1b) The daughter cell joins the group of its parent. Group formation via CT is implemented by allowing sticky solitary cells to join groups or other solitary cells with probability $\beta$ at each time step. We do not consider CT events between groups of size larger than 1 . Cells dissociate from their group with probability $\left(1-s_{\text {cell }}\right)$ $\left(1-s_{\text {group }}\right)$ at each time step, where $s_{\text {cell }}$ is the stickiness of the cell and $s_{\text {group }}$ is the mean stickiness of all group members (Fig. 1b). Cells that are not sticky $\left(s_{\text {cell }}=0\right)$ are more likely to leave their group than cells that are sticky $\left(s_{\text {cell }}=s\right)$. In particular, a group that has only non-sticky members $\left(s_{\text {group }}=0\right)$ falls apart instantaneously because every group member has dissociation probability 1 . This means that non-sticky cells are not able to form groups by ST, because, after division, the two daughter cells will separate immediately. Groups always disintegrate cell-by-cell; we do not consider group fragmentation.

Costs and benefits of group formation. The minimal group size required to avoid predation is denoted by $k$. At each time step, groups of size lower than $k$ die as a 
whole, due to predation, with probability $d_{\text {pred }}$ (same as solitary cells). Solitary cells, as well as cells within groups (including those of size at least $k$ ), undergo intrinsic cell death with probability $d_{\text {cell }}$. The costs of group formation are implemented by dividing the division probability $b$ (defined above) of each cell in a group by the size of the group. Thus, the combined reproductive potential of all cells in a group equals the reproductive potential of a solitary cell.

Reporting Summary. Further information on research design is available in the Nature Research Reporting Summary linked to this article.

\section{Data availability}

The study is theoretical; no new empirical data were generated.

\section{Code availability}

The simulation code supporting this work is available for download from https:// doi.org/10.5281/zenodo.2845406. Pseudocode is available in the Supplementary Methods.

Received: 17 December 2018; Accepted: 4 June 2019;

Published online: 8 July 2019

\section{References}

1. Buss, L. W. The Evolution of Individuality (Princeton Univ. Press, 1987)

2. van Gestel, J. \& Tarnita, C. E. On the origin of biological construction, with a focus on multicellularity. Proc. Natl Acad. Sci. USA 114, 11018-11026 (2017).

3. Maynard Smith, J. \& Szathmary, E. The Major Transitions in Evolution (Oxford Univ. Press, 1997).

4. West, S. A., Fisher, R. M., Gardner, A. \& Kiers, E. T. Major evolutionary transitions in individuality. Proc. Natl Acad. Sci. USA 112, 10112-10119 (2015)

5. Szathmáry, E. Toward major evolutionary transitions theory 2.0. Proc. Natl Acad. Sci. USA 112, 10104-10111 (2015).

6. Grosberg, R. K. \& Strathmann, R. R. The evolution of multicellularity: a minor major transition? Annu. Rev. Ecol. Evol. Syst. 38, 621-654 (2007)

7. De Monte, S. \& Rainey, P. B. Nascent multicellular life and the emergence of individuality. J. Biosci. 39, 237-248 (2014).

8. Rainey, P. B. \& De Monte, S. Resolving conflicts during the evolutionary transition to multicellular life. Annu. Rev. Ecol. Evol. Syst. 45, 599-620 (2014).

9. Bonner, J. T. The origins of multicellularity. Integr. Biol. 1, 27-36 (1998).

10. Bonner, J. T. Size and Cycle: An Essay on the Structure of Biology (Princeton Univ. Press, 1965).

11. Claessen, D., Rozen, D. E., Kuipers, O. P., Søgaard-Andersen, L. \& van Wezel, G. P. Bacterial solutions to multicellularity: a tale of biofilms, filaments and fruiting bodies. Nat. Rev. Microbiol. 12, 115-124 (2014).

12. Coelho, S. M. et al. Complex life cycles of multicellular eukaryotes: new approaches based on the use of model organisms. Gene 406, 152-170 (2007).

13. Herron, M. D., Rashidi, A., Shelton, D. E. \& Driscoll, W. W. Cellular differentiation and individuality in the 'minor' multicellular taxa. Biol. Rev. 88, 844-861 (2013).

14. Lyons, N. A. \& Kolter, R. On the evolution of bacterial multicellularity. Curr. Opin. Microbiol. 24, 21-28 (2015).

15. Griesemer, J. Reproduction in complex life cycles: toward a developmental reaction norms perspective. Philos. Sci. 83, 803-815 (2016).

16. Libby, E. \& Rainey, P. B. A conceptual framework for the evolutionary origins of multicellularity. Phys. Biol. 10, 035001 (2013).

17. Nanjundiah, V., Kirk, D. \& Ruiz-Trillo, I. in Cells in Evolutionary Biology (eds. Hall, B. K. \& Moody, S. A.) Ch. 4 (CRC Press, 2018).

18. Bonner, J. T. Life Cycles: Reflections of an Evolutionary Biologist (Princeton Univ. Press, 1993).

19. Tarnita, C. E., Taubes, C. H. \& Nowak, M. A. Evolutionary construction by staying together and coming together. J. Theor. Biol. 320, 10-22 (2013).

20. Bonner, J. T. Cells and Societies (Princeton Univ. Press, 1955).

21. Pichugin, Y., Peña, J., Rainey, P. B. \& Traulsen, A. Fragmentation modes and the evolution of life cycles. PLoS Comp. Biol. 13, e1005860 (2017).

22. Roze, D. \& Michod, R. E. Mutation, multilevel selection, and the evolution of propagule size during the origin of multicellularity. Am. Nat. 158, 638-654 (2001).

23. Grosberg, R. K. \& Strathmann, R. R. One cell, two cell, red cell, blue cell: the persistence of a unicellular stage in multicellular life histories. Trends Ecol. Evol. 13, 112-116 (1998).

24. King, N. The unicellular ancestry of animal development. Dev. Cell 7, 313-325 (2004).

25. Sebé-Pedrós, A., Degnan, B. M. \& Ruiz-Trillo, I. The origin of metazoa: a unicellular perspective. Nat. Rev. Genet. 18, 498-512 (2017).

26. Brunet, T. \& King, N. The origin of animal multicellularity and cell differentiation. Dev. Cell 43, 124-140 (2017).

27. Suga, H. \& Ruiz-Trillo, I. Development of ichthyosporeans sheds light on the origin of metazoan multicellularity. Dev. Biol. 377, 284-292 (2013).
28. Sebé-Pedrós, A. et al. Regulated aggregative multicellularity in a close unicellular relative of metazoa. eLife 2, e01287 (2013).

29. Sogabe, S. et al. Pluripotency and the origin of animal multicellularity. Nature https://doi.org/10.1038/s41586-019-1290-4 (2019).

30. Hammerschmidt, K., Rose, C. J., Kerr, B. \& Rainey, P. B. Life cycles, fitness decoupling and the evolution of multicellularity. Nature 515, 75-79 (2014).

31. Ratcliff, W. C., Denison, R. F., Borrello, M. \& Travisano, M. Experimental evolution of multicellularity. Proc. Natl Acad. Sci. USA 109, 1595-1600 (2012).

32. Ratcliff, W. C. et al. Experimental evolution of an alternating uni- and multicellular life cycle in Chlamydomonas reinhardtii. Nat. Commun. $\mathbf{4}$, 2742 (2013)

33. Koschwanez, J. H., Foster, K. R. \& Murray, A. W. Sucrose utilization in budding yeast as a model for the origin of undifferentiated multicellularity. PLoS Biol. 9, e1001122 (2011)

34. Herron, M. D. et al. De novo origins of multicellularity in response to predation. Sci. Rep. 9, 2328 (2019).

35. Hanschen, E. R. et al. The Gonium pectorale genome demonstrates co-option of cell cycle regulation during the evolution of multicellularity. Nat. Commun. 7, 11370 (2016)

36. King, N., Hittinger, C. T. \& Carroll, S. B. Evolution of key cell signaling and adhesion protein families predates animal origins. Science 301, 361-363 (2003).

37. Rokas, A. The molecular origins of multicellular transitions. Curr. Opin. Genet. Dev. 18, 472-478 (2008).

38. Sebé-Pedrós, A. et al. The dynamic regulatory genome of Capsaspora and the origin of animal multicellularity. Cell 165, 1224-1237 (2016).

39. Rossetti, V., Filippini, M., Svercel, M., Barbour, A. D. \& Bagheri, H. C. Emergent multicellular life cycles in filamentous bacteria owing to densitydependent population dynamics. J. R. Soc. Interface 8, 1772-1784 (2011).

40. Furusawa, C. \& Kaneko, K. Origin of multicellular organisms as an inevitable consequence of dynamical systems. Anat. Rec. 268, 327-342 (2002).

41. van Gestel, J. \& Nowak, M. A. Phenotypic heterogeneity and the evolution of bacterial life cycles. PLoS Comp. Biol. 12, e1004764 (2016).

42. Pichugin, Y. \& Traulsen, A. Reproduction costs can drive the evolution of groups. Preprint at bioRxiv https://doi.org/10.1101/325670 (2018).

43. Rashidi, A., Shelton, D. E. \& Michod, R. E. A Darwinian approach to the origin of life cycles with group properties. Theor. Popul. Biol. 102 76-84 (2015).

44. Gao, Y., Traulsen, A. \& Pichugin, Y. Interacting cells driving the evolution of multicellular life cycles. PLoS Comp. Biol. 15, e1006987 (2019).

45. Ratcliff, W. C., Herron, M., Conlin, P. L. \& Libby, E. Nascent life cycles and the emergence of higher-level individuality. Philos. Trans. R. Soc. B 372, 20160420 (2017)

46. Willensdorfer, M. On the evolution of differentiated multicellularity. Evolution 63, 306-323 (2009).

47. Gavrilets, S. Rapid transition towards the division of labor via evolution of developmental plasticity. PLoS Comp. Biol. 6, e1000805 (2010).

48. Ispolatov, I., Ackermann, M. \& Doebeli, M. Division of labour and the evolution of multicellularity. Proc. R. Soc. B. 279, 1768-1776 (2012).

49. Garcia, T., Brunnet, L. G. \& De Monte, S. Differential adhesion between moving particles as a mechanism for the evolution of social groups. PLoS Comp. Biol. 10, e1003482 (2014).

50. Garcia, T., Doulcier, G. \& De Monte, S. The evolution of adhesiveness as a social adaptation. eLife 4, e08595 (2015).

51. Kauffman, S. A. Metabolic stability and epigenesis in randomly constructed genetic nets. J. Theor. Biol. 22, 437-467 (1969).

52. Davidson, E. H. The Regulatory Genome: Gene Regulatory Networks In Development And Evolution (Academic Press, 2006).

53. Karlebach, G. \& Shamir, R. Modelling and analysis of gene regulatory networks. Nat. Rev. Mol. Cell Biol. 9, 770-780 (2008).

54. Smith, S. J., Rebeiz, M. \& Davidson, L. From pattern to process: studies at the interface of gene regulatory networks, morphogenesis, and evolution. Curr. Opin. Genet. Dev. 51, 103-110 (2018).

55. Aharoni, A. et al. The 'evolvability' of promiscuous protein functions. Nat. Genet. 37, 73-76 (2005).

56. Piatigorsky, J. Gene Sharing and Evolution: The Diversity of Protein Functions (Harvard Univ. Press, 2009).

57. Piatigorsky, J. et al. Gene sharing by delta-crystallin and argininosuccinate lyase. Proc. Natl Acad. Sci. USA 85, 3479-3483 (1988).

58. Nedelcu, A. M. \& Michod, R. E. The evolutionary origin of an altruistic gene. Mol. Biol. Evol. 23, 1460-1464 (2006).

59. Ritchie, A. V., van Es, S., Fouquet, C. \& Schaap, P. From drought sensing to developmental control: evolution of cyclic AMP signaling in social amoebas. Mol. Biol. Evol. 25, 2109-2118 (2008).

60. Abedin, M. \& King, N. Diverse evolutionary paths to cell adhesion. Trends Cell Biol. 20, 734-742 (2010).

61. Abedin, M. \& King, N. The premetazoan ancestry of cadherins. Science 319, 946-948 (2008) 
62. Boraas, M. E., Seale, D. B. \& Boxhorn, J. E. Phagotrophy by a flagellate selects for colonial prey: a possible origin of multicellularity. Evol. Ecol. 12, 153-164 (1998).

63. Smukalla, S. et al. FLO1 is a variable green beard gene that drives biofilm-like cooperation in budding yeast. Cell 135, 726-737 (2008).

64. Pianka, E. R. On r- and K-selection. Am. Nat. 104, 592-597 (1970).

65. MacArthur, R. H. \& Wilson, E. O. The Theory of Island Biogeography (Princeton Univ. Press, 1967).

66. Avilés, L., Fletcher, J. A., Cutter, A. D. \& Perrin, A. E. N. The kin composition of social groups: trading group size for degree of altruism. Am. Nat. 164, 132-144 (2004).

67. Pentz, J. T., Márquez-Zacarías, P., Yunker, P. J., Libby, E. \& Ratcliff, W. C. Ecological advantages and evolutionary limitations of aggregative multicellular development. Preprint at bioRxiv https://doi.org/10.1101/255307 (2018)

68. Libby, E. et al. Geometry shapes evolution of early multicellularity. PLoS Computat. Biol. 10, e1003803 (2014).

69. Joshi, J., Couzin, I. D., Levin, S. A. \& Guttal, V. Mobility can promote the evolution of cooperation via emergent self-assortment dynamics. PLoS Comp. Biol. 13, e1005732 (2017).

70. Libby, E. \& Rainey, P. B. Eco-evolutionary feedback and the tuning of proto-developmental life cycles. PLoS ONE 8, e82274 (2013).

71. Niklas, K. J. The evolutionary-developmental origins of multicellularity. Am. J. Bot. 101, 6-25 (2014).

72. Minelli, A. \& Fusco, G. Developmental plasticity and the evolution of animal complex life cycles. Philos. Trans. R. Soc. B 365, 631-640 (2010).

73. Wolpert, L. \& Szathmáry, E. Multicellularity: evolution and the egg. Nature 420, 745 (2002).

74. Michod, R. E. Evolution of individuality during the transition from unicellular to multicellular life. Proc. Natl Acad. Sci. USA $\mathbf{1 0 4}$ 8613-8618 (2007).

75. Shapiro, J. A. Thinking about bacterial populations as multicellular organisms. Annu. Rev. Microbiol. 52, 81-104 (1998).

76. McDougald, D., Rice, S. A., Barraud, N., Steinberg, P. D. \& Kjelleberg, S. Should we stay or should we go: mechanisms and ecological consequences for biofilm dispersal. Nat. Rev. Microbiol. 10, 39-50 (2012).

77. Schaap, P. Evolutionary crossroads in developmental biology: Dictyostelium discoideum. Development 138, 387-396 (2011).

78. West, S. A., Diggle, S. P., Buckling, A., Gardner, A. \& Griffin, A. S. The social lives of microbes. Annu. Rev. Ecol. Evol. Syst. 38, 53-77 (2007).

79. Brown, M. W., Kolisko, M., Silberman, J. D. \& Roger, A. J. Aggregative multicellularity evolved independently in the eukaryotic supergroup Rhizaria. Curr. Biol. 22, 1123-1127 (2012).

80. Queller, D. C. \& Strassmann, J. E. Beyond society: the evolution of organismality. Philos. Trans. R. Soc. B 364, 3143-3155 (2009).

81. Gadagkar, R. \& Bonner, J. Social insects and social amoebae. J. Biosci. 19, 219-245 (1994).
82. Quiñones, A. E. \& Pen, I. A unified model of Hymenopteran preadaptations that trigger the evolutionary transition to eusociality. Nat. Commun. 8 , 15920 (2017).

83. Wilson, E. O. The Insect Societies (Belknap Press of Harvard Univ. Press, 1971).

84. Riehl, C. Evolutionary routes to non-kin cooperative breeding in birds. Proc R. Soc. B 280, 20132245 (2013).

85. Cockburn, A. Evolution of helping behavior in cooperatively breeding birds. Annu. Rev. Ecol. Syst. 29, 141-177 (1998).

86. Sherman, P. W., Lacey, E. A., Reeve, H. K. \& Keller, L. Forum: The eusociality continuum. Behav. Ecol. 6, 102-108 (1995).

87. Alexander, R. D. The evolution of social behavior. Annu. Rev. Ecol. Syst. 5, 325-383 (1974)

88. Bourke, A. F. G., Franks, N. R. \& Franks, N. R. Social Evolution in Ants (Princeton Univ. Press, 1995).

89. Gordon, D. M. Ant Encounters: Interaction Networks and Colony Behavior (Princeton Univ. Press, 2010).

90. Wilson, E. B. Probable inference, the law of succession, and statistical inference. J. Am. Stat. Assoc. 22, 209-212 (1927).

\section{Acknowledgements}

We dedicate this study to the memory of John Tyler Bonner, whose work has been a source of great inspiration. We thank S. De Monte for comments and discussion and the Theoretical Biology \& Bioinformatics group at Utrecht University for computing resources. J.v.G. received support from the EMBO Long-Term Fellowship (no. ALTF 1101-2016) and the Marie Sklodowska-Curie Individual Fellowship (no. 742235). C.E.T. acknowledges support from the US National Science Foundation (no. RoL:FELS:EAGER\#1838331).

\section{Author contributions}

J.v.G. and C.E.T. conceived the study. All authors developed the model. M.S. performed the computational work and analysed the data. All authors interpreted the results and wrote the manuscript.

\section{Competing interests}

The authors declare no competing interests.

\section{Additional information}

Supplementary information is available for this paper at https://doi.org/10.1038/ s41559-019-0940-0.

Reprints and permissions information is available at www.nature.com/reprints. Correspondence and requests for materials should be addressed to J.v. or C.E.T. Publisher's note: Springer Nature remains neutral with regard to jurisdictional claims in published maps and institutional affiliations.

(c) The Author(s), under exclusive licence to Springer Nature Limited 2019 


\section{Reporting Summary}

Nature Research wishes to improve the reproducibility of the work that we publish. This form provides structure for consistency and transparency in reporting. For further information on Nature Research policies, see Authors \& Referees and the Editorial Policy Checklist.

\section{Statistics}

For all statistical analyses, confirm that the following items are present in the figure legend, table legend, main text, or Methods section.

n/a $\mid$ Confirmed

$\square$ \. $\square$ The exact sample size $(n)$ for each experimental group/condition, given as a discrete number and unit of measurement

Х $\square$ A statement on whether measurements were taken from distinct samples or whether the same sample was measured repeatedly

$\square$ The statistical test(s) used AND whether they are one- or two-sided

$\triangle$ Only common tests should be described solely by name; describe more complex techniques in the Methods section.

Х $\square$ A description of all covariates tested

Х $\square$ A description of any assumptions or corrections, such as tests of normality and adjustment for multiple comparisons

\ $\square$ A full description of the statistical parameters including central tendency (e.g. means) or other basic estimates (e.g. regression coefficient)

$\triangle \square$ AND variation (e.g. standard deviation) or associated estimates of uncertainty (e.g. confidence intervals)

$\square$ For null hypothesis testing, the test statistic (e.g. $F, t, r$ ) with confidence intervals, effect sizes, degrees of freedom and $P$ value noted

$\bigotimes \square$ Give $P$ values as exact values whenever suitable.

Х $\square$ For Bayesian analysis, information on the choice of priors and Markov chain Monte Carlo settings

Х $\square$ For hierarchical and complex designs, identification of the appropriate level for tests and full reporting of outcomes

Х $\square$ Estimates of effect sizes (e.g. Cohen's $d$, Pearson's $r$ ), indicating how they were calculated

Our web collection on statistics for biologists contains articles on many of the points above.

\section{Software and code}

Policy information about availability of computer code

Data collection Custom Python code was used to run simulations. The code will be made publicly available.

Data analysis Custom Python and $\mathrm{R}$ code was used to analyze the data.

For manuscripts utilizing custom algorithms or software that are central to the research but not yet described in published literature, software must be made available to editors/reviewers. We strongly encourage code deposition in a community repository (e.g. GitHub). See the Nature Research guidelines for submitting code \& software for further information.

\section{Data}

Policy information about availability of data

All manuscripts must include a data availability statement. This statement should provide the following information, where applicable:

- Accession codes, unique identifiers, or web links for publicly available datasets

- A list of figures that have associated raw data

- A description of any restrictions on data availability

The study is theoretical; no new empirical data were generated.

\section{Field-specific reporting}

Please select the one below that is the best fit for your research. If you are not sure, read the appropriate sections before making your selection.
Life sciences
$\square$ Behavioural \& social sciences
$\bigotimes$ Ecological, evolutionary \& environmental sciences

For a reference copy of the document with all sections, see nature.com/documents/nr-reporting-summary-flat.pdf 


\section{Ecological, evolutionary \& environmental sciences study design}

All studies must disclose on these points even when the disclosure is negative.

Study description The emergence of multicellular life cycles during an evolutionary transition to multicellularity was studied. The study was conducted using simulations implemented in Python.

Research sample All data were generated by running simulations of the computational model. For each parameter combination, multiple replicate simulations were run.

Sampling strategy

Multiple replicate simulations were run for each parameter combination (at least 10). No statistical methods were used to determine the number of simulations.

Data collection

All data were collected from the simulations. Abundance of different strategies in the simulations was evaluated every 7,500 time steps.

Timing and spatial scale

Each simulation was run for at least 1,000,000 time steps (at least 1,000 generations). The model is not spatially explicit.

Data exclusions

No data were excluded from the analyses. For visualization purposes, some figures only show a (randomly chosen) subset of the data (e.g. results for only $9 / 10$ simulations are shown in Fig. 2 and 5.)

Reproducibility

Not applicable as all data were generated using computer simulations.

Randomization

Not applicable as all data were generated using computer simulations.

Blinding

Blinding was not performed as the data were analyzed algorithmically rather than manually; results for each simulation were processed using the same computational pipeline.

Did the study involve field work? $\square$ Yes $\quad$ No

\section{Reporting for specific materials, systems and methods}

We require information from authors about some types of materials, experimental systems and methods used in many studies. Here, indicate whether each material, system or method listed is relevant to your study. If you are not sure if a list item applies to your research, read the appropriate section before selecting a response.

\begin{tabular}{|c|c|}
\hline$n / a$ & Involved in the study \\
\hline Х & Antibodies \\
\hline Х & Eukaryotic cell lines \\
\hline Х & Palaeontology \\
\hline Х & Animals and other organisms \\
\hline Х & Human research participants \\
\hline Х & Clinical data \\
\hline
\end{tabular}

\begin{tabular}{l|l}
\multicolumn{2}{l}{ Methods } \\
\hline n/a & Involved in the study \\
$\bigotimes$ & $\square$ ChIP-seq \\
$\triangle$ & $\square$ Flow cytometry \\
$\square$ & $\square$ MRI-based neuroimaging
\end{tabular}

